\title{
Transdermal Alcohol Measurements Using MOX Sensors in Clinical Trials ${ }^{\dagger}$
}

\author{
Bruno Lawson 1, Virginie Martini-Laithier 1, Tomas Fiorido 1, Fatima Annanouch 1, \\ Stephane Burtey ${ }^{2}$, Catherine Cassé-Perrot ${ }^{3}$, Christine Audebert ${ }^{3}$, Marc Bendahan ${ }^{1}$, \\ Rachid Bouchakour ${ }^{1}$, Olivier Blin ${ }^{3}$ and Khalifa Aguir 1,* \\ 1 Aix Marseille University, Université de Toulon, CNRS, IM2NP, Marseille, France; \\ bruno.lawson@im2np.fr (B.L.); virginie.laithier@im2np.fr (V.M.-L.); tomas.fiorido@im2np.fr (T.F.); \\ fatima.annanouch@im2np.fr (F.A.); marc.bendahan@im2np.fr (M.B.); rachid.bouchakour@im2np.fr (R.B.) \\ 2 Aix Marseille University, INSERM UMR_S 1076 Marseille, France; stephane.burtey@univ-amu.fr \\ 3 Aix Marseille University, Pharmacologie Clinique, AP-HM, Marseille, France; \\ Catherine.PERROT@ap-hm.fr (C.C.-P.); Christine.AUDEBERT@ap-hm.fr (C.A.); Olivier.BLIN@ap-hm.fr (O.B.) \\ * Correspondence: khalifa.aguir@im2np.fr; Tel.: +33-491-288-972 \\ + Presented at the Eurosensors 2017 Conference, Paris, France, 3-6 September 2017.
}

Published: 11 August 2017

\begin{abstract}
Human metabolism often results in the emission of many VOCs through the skin. Ethanol is one of volatile compounds which are evaporated by perspiration. The aim of our research consists to develop chemical sensors for monitoring ethanol emission after alcohol consumption. The interest of using chemical sensors is noninvasive measurement and controlling alcohol level in the human body and to make the link between these measurements and that in the blood or in the breath. Recent clinical trials demonstrated the feasibility and relevance of this measurement method. Metal oxide sensors were calibrated in respect of the thermodynamic conditions of the surface of the skin. In this paper we show the first sensor responses by perspiration.
\end{abstract}

Keywords: gas sensor; VOCs; ethanol detection; perspiration; transdermal alcohol; alcohol pharmacokinetics

\section{Introduction}

Since several decades the increasing of means for human lives safety, created great interest in research field of volatile organic compounds (VOCs) detection in human body. The literature reported up to 400 VOCs emitted by human skin. Some of these can be identified as disease biomarkers [1]. Ethanol is one of the VOCs found in skin perspiration. Alcohol consumption generates the increase of ethanol concentration emitted by skin. After ethanol ingestion, it is metabolized principally by the liver and a little part is removed by exhaled air or breath and also through the skin in proportion of $1 \%$ of consumed alcohol [2,3]. Alcohol is one of the major preoccupations of our century, causing thousands of deaths every year and representing a very high socio-economic cost. In order to fight this and make detection easier, we need non-invasive practical methods to account and measure the Blood Alcohol Concentration (BAC) continuously with reliability. At present, there are some techniques for the measurement of blood alcohol levels via blood, urine, saliva, sweat or exhaled air analysis. The most commonly used techniques are firstly blood analysis, which requires an invasive test with syringe and need professional assistance. Secondly exhaled air measurements, contrary to perspiration can only be carried out non-continuously and need sample collection. Our work consists to detect ethanol in perspiration by using metal oxide (MOX)-based gas microsensors which are interest by its small size, low power consumption, continuous and noninvasive measurements, good sensitivity and short response time 
and can be integrated in embedded and autonomous device. In this study, we monitored continuously transdermal alcohol concentration (TAC) with MOX microsensors and we correlated these data with blood (BAC) and breath (BrAC) alcohol concentration, during clinical tests. To achieve our aims we selected 3 most sensitive commercial MOX microsensors which have been integrated in electronic bracelet for clinical trials.

\section{Experimental Set Up}

An innovative and automated sensor system calibration was designed and built thanks to the APPRISE (Personal Assistant of Health Prevention) project in order to test and study the sensor performances under ethanol exposures. The experimental set up (Figure 1) is made of three parts: generation bench of controlled ethanol vapor. Ethanol vapor is generated by permeation system with different stages of dilutions via several automated mass flow controllers (Figure 1a). Ethanol concentrations have been studied between $1 \mathrm{ppm}$ and $100 \mathrm{ppm}$ with a relative humidity of $50 \%-\mathrm{An}$ environmental chamber with temperature and humidity control with an integrated test cell made in our laboratory to characterize and to calibrate the sensors in the presence of various ethanol concentrations (Figure 1b) - A control and data acquisition system (Figure 1c)

The gas flow rate is fixed at $250 \mathrm{sccm}$. Ethanol vapor is carried in the test chamber placed in the environmental chamber at $33{ }^{\circ} \mathrm{C}$ which corresponds to the average temperature of the skin surface. The sensor resistances are measured directly by three Keithley 2450 sourcemeters with a constant current bias at $1.7 \mu \mathrm{A}$.

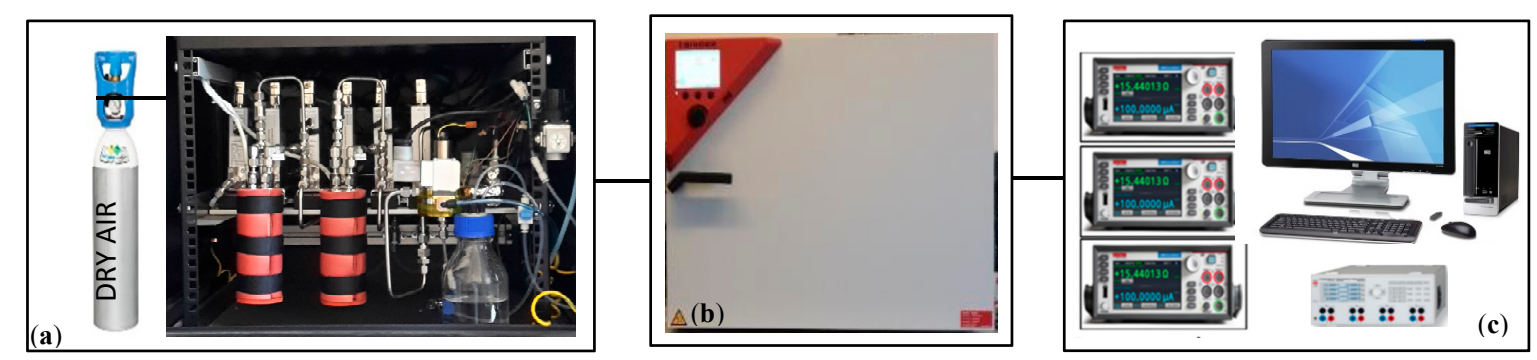

Figure 1. Experimental set-up for ethanol microsensors characterization and calibration: (a) ethanol generation bench, (b) environmental chamber, (c) control and data acquisition system.

\section{Sensors Calibration in the Laboratory Test Bench}

The three commercial VOCs sensors were selected according to ethanol sensitivity and power consumption: MICS 5524 (SGX Sensors), TGS 8100 (FIGARO Sensors) and CCS 801 (Cambridge Sensors). The sensors were exposed to different ethanol concentrations during $30 \mathrm{~s}$ from $1 \mathrm{ppm}$ to $100 \mathrm{ppm}$ with $50 \%$ relative humidity at a flow rate of $250 \mathrm{sccm}$. The MICS 5524 sensor showed the best sensitivity (Figure 2) under ethanol vapor and relative humidity rate (HR 50\%). Therefore, we have chosen to present only the results corresponding to the sensor MICS 5524 during the clinical trials.



Figure 2. Sensor resistance variation for exposition to different ethanol concentrations. 


\section{Clinical Trials}

\subsection{Aim}

This part of the work was conducted at the CIC-CPCET (Centre d'Investigation CliniquesCentre de Pharmacologie Clinique et d'Evaluations Thérapeutiques) of the Timone University hospital at the Assistance Publique of Marseille, thanks to Apprise project. The first objective is to prove the detection of ethanol in the perspiration by MOX-based sensors. The second is to study the kinetics of alcohol metabolism in blood and exhaled air and the kinetics of alcohol diffusion through the skin in order to estimate the cutaneous clearance of alcohol. Medical team was in charge of selection of volunteers according to inclusion criteria defined by the protocol (age between 18 and 40, caucasian, alcohol consumption between 3 to 14 standard glasses/week, BMI between 19 to $28 \mathrm{~kg} / \mathrm{m}^{2}$, no smoker or $<5 \mathrm{cig} /$ day) and of medical supervision. Their rules consist also to analyze blood and breath of volunteers before and after alcohol consumption. Some others medical and psychological tests were made to complete the analysis. In order to obtain reliable and homogeneous data during this clinical trial, 6 subjects were selected, consisting of 3 females and 3 males.

\subsection{Clinical Protocol}

This protocol was approved by Ethic Committee and ANSM (National Agency for Safety of medicines and health Products) and conducted according to Good Clinical Practices and all subjects gave written informed consent before entering the trial. During this clinical trial two doses of alcohols in the body will be studied, BAC of $0.5 \mathrm{~g} / \mathrm{L}$ and $0.8 \mathrm{~g} / \mathrm{L}$; In compliance with the limits laid down by French legislation. The amount of alcohol consumed by each subject (A glass of a mixture of orange juice-alcohol, $40 \%$ vodka) is calculated by the widmark formula which is not absolute and therefore may have some variability. Following administration of the mixture containing the defined quantity of alcohol, different samples were taken simultaneously at precise times $\left(0,15^{\prime}, 30^{\prime}, 45^{\prime}, 60^{\prime}, 1 \mathrm{~h} \mathrm{30}\right.$, $2 \mathrm{~h}, 2 \mathrm{~h} \mathrm{30}$, $3 \mathrm{~h}, 4 \mathrm{~h}, 6 \mathrm{~h}, 8 \mathrm{~h}$, and $10 \mathrm{~h}$ ) : Blood samples (BAC), Breath samples (BrAC) and conductance samples (TAC, Bracelet three MOX Sensors). For the clinical trials, a specific bracelet (Figure 3) and a mobile test bench (Figure 4) have been specially designed. The gas detection bracelet is composed of a circuit of 3 gas sensors on a PCB integrated in a little cavity. The cavity is opened on the top of the wrist skin surface. The surface of the opened area is around $5 \mathrm{~cm}^{2}$ and the distance between gas sensors and the skin is $3 \mathrm{~mm}$.

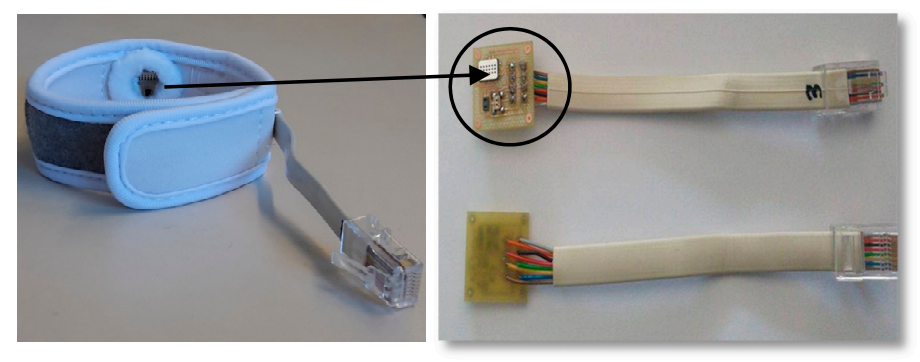

Figure 3. Bracelet with the three MOX sensors.

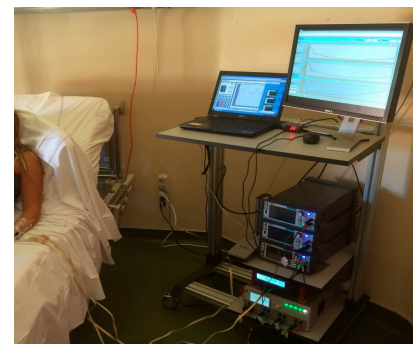

Figure 4. Mobile test bench for clinical trials. 


\subsection{Results and Discussion}

Figure 5 shows the evolution of BAC, BrAC and TAC as a function of time for a person who consumed the equivalent of $0.8 \mathrm{~g}$ of alcohol per liter in the blood. After drinking alcohol, blood tests and expiration tests showed a rapid rise in blood alcohol level to a maximum of about 45 min afterwards. It takes about $6 \mathrm{~h}$ to stop traces of alcohol in the blood.

In the same way we will note an increase in the conductance of the MOX sensors, followed by a slow decrease cf. Figure 5. This figure shows only one result, note that the results of the other sensors and of the others volunteers are comparable. This variation can therefore be correlated with the concentration of alcohol in the blood. These results show that ethanol evaporated from the skin is in sufficient quantity to be detected by MOX sensors by perspiration and the blood alcohol level (BAC) can be estimated after sensors calibration. We can also observed that the TAC alcohol curve $(G)$ is right shifted from the BrAC and BAC alcohol curves and takes longer time to reach zero. The same kinetic behavior was observed in alcohol elimination by urine [4]. This point will be discussed.

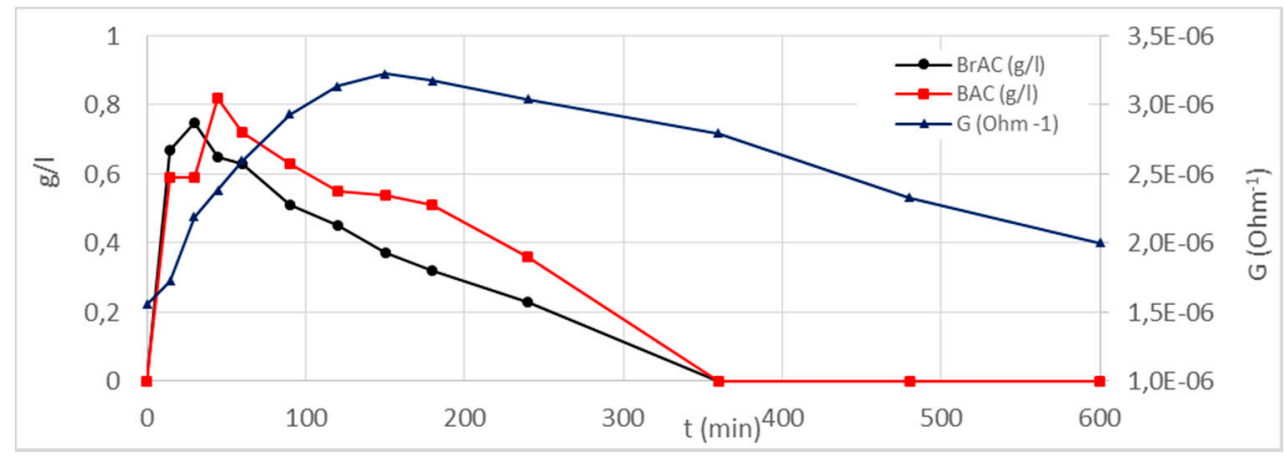

Figure 5. Evolution $\mathrm{BAC}, \mathrm{BrAC}$ and $\mathrm{TAC}$ as a function of time for a subject who consumed the equivalent of $0.8 \mathrm{~g}$ of alcohol per liter in the blood.

\section{Conclusions}

This paper reports evidence that it is possible to detect ethanol vapor through perspiration across the skin using MOX sensors. The results are in agreement with the two concentrations of ethanol absorbed by the volunteers. The responses are higher for the $0.8 \mathrm{~g} / \mathrm{L}$ dose and are slightly shifted in time because of metabolic processes. This shift is slightly less pronounced among women. On the other hand it can be noted that it is possible to detect ethanol at longer times than with other techniques.

Acknowledgments: We wish to thank AMU-AMIDEX for their financial support. We also thanks Alain COMBES for his technical support throughout this work.

Conflicts of Interest: The authors declare no conflict of interest.

\section{References}

1. Mochalski, P.; King, J. Emission rates of selected volatile organic compounds from skin of healthy volunteers. J. Chromatogr. B 2014, 959, 62-70

2. Paul, R.M.; McKnight, A.S. Evaluation Transdermal Alcohol Measuring Device; Pacific Institute for research and Evaluation: Calverton, NY, USA, 2007.

3. Widmark, E.M.P. Principles and Applications of Medico Legal Alcohol Determination; English translation of 1932 German ed.; Davis Biomedical Publications: Davis, CA, USA, 1981.

4. Batt, R.D. Adsorption, distribution and elimination of alcohol. In Human Metabolism of Alcohol; Crow, K.E., Batt, R.D., Eds.; CRC Press: Boca Raton, FL, USA, 1989; Volume I. 